\title{
Nutrition, Diets, Feeding, and Swallowing Management in Public Schools
}

\author{
Stephen Enwefa, Regina Enwefa \\ Southern University and A \& M College, Baton Rouge, LA USA
}

\begin{abstract}
Nutrition is a gateway for preventing diseases in an effort to promote good health. Lack of adequate nutrition produces structural damage to the brain. Feeding involves the specific act of food preparation and getting the food to the child orally or through other means. Swallowing is the inability or difficulty moving food from the mouth to the stomach by individuals [6]. The goals of appropriate nutrition, diets, feeding, and swallowing management are to prevent diseases, promote hydration, and normal calorie intake that lead to optimum health and wellness. Several studies have reported the prevalence and incidence of swallowing disorders in public schools [6], [8], [9], [10]. Approximately, $25 \%-35 \%$ of normally developed children have pediatric feeding and swallowing disorders [6], [8]. It has been reported that $25 \%-45 \%$ of typically developing children demonstrate feeding and swallowing problems [6], [8, [9], [10]. The prevalence is estimated to be between $30 \%$ to $80 \%$ for children with developmental disorders [5]. From birth to school age, children experience swallowing complications, feeding and mealtime issues which overtime can lead to nutritional challenges for them. This study examined nutrition, diets, feeding and swallowing management in public schools; described swallowing and feeding challenges, who is at risk, swallowing and feeding dietary modifications, and interdisciplinary team assessment/management. Furthermore, the study discussed cultural and linguistic diversity for swallowing and feeding considerations, strategies for feeding and swallowing problems, accommodations and modifications for children, physician medical statement for services, and requiring a child's medical statement for local education agencies. Finally, the study highlighted nutrition, swallowing, and feeding needs.
\end{abstract}

\section{Introduction}

Nutrition is a gateway for preventing diseases in an effort to promote good health. Lack of adequate nutrition produces structural damage to the brain. Over the last several years about 14 million children or more in the United States had diets that were significantly below the recommended allowance for body nutrients. Socioeconomic status is the most powerful predictor of disease, disorder, injury and mortality [8], [10]. The 21 st century society must shift the focus of healthcare away from how to treat diseases and disorders to acknowledging the reasons why diseases and disorders manifest in the first place. Swallowing has several definitions and one of the most frequently used is difficulty moving food from the mouth to the stomach. More recently, clinicians have expanded the definition to include: behavioral, sensory and motor acts in preparation for swallowing to include cognitive awareness of eating situations, visual recognition of food, food diary, physiologic responses to smell and/or presence of food that increases salivation. [8], [9], [10].

Increasingly, swallowing disorders can occur as a result of a variety of congenital anomalies, structural damage, and medical conditions in all age groups. Feeding problems have been recognized in clinical literature for over half a century. Children experiencing feeding difficulty exhibit signs of refusal to eat orally and/or inability to sustain oral feedings to maintain adequate calorie intake for good health. Common etiologies associated with feeding problems in children are: sensory deprivation, anatomical anomalies, social and behavioral maladaptation, gastrointestinal diseases/dysfunction, cardio respiratory compromise, and neurological dysfunction. Children within today's public schools are faced with a multitude of problems relative to swallowing, feeding, diet and nutrition. Whenever a person has a feeding, swallowing disorder, and lack of nutrition, it is important to understand the necessity for hydration and nutrition for academic success. Before gaining insights to swallowing, feeding, and nutrition, one must be knowledgeable about the anatomical and physiological aspects, stages of swallowing, prevalence and incidence of swallowing, prevalence and incidence of feeding, challenges to nutrition, diets, and etiological factors.

Presently, there is lack of empirical data as to a defined best practice plan for swallowing professionals. This is partly due to methodological issues in most of the previous studies. The results of majorities of the studies indicated the need for streamlining the referral process, team members' composition, assessment, and treatment processes. For many years, speech language pathologists have been involved in the evaluation and treatment of individuals with swallowing disorders. The necessity, demand, and increase for speech language pathologist involvement with dysphagia management has increased tremendously, and major growth spurts 
within the profession is quite enormous. [16] described that the scope of practice for dysphagia services has long been deliberated within the school setting that swallowing management was practiced only in the medical setting. Due to the advancements in medical technology and access to care, many children who are born with etiological factors resulting in swallowing problems are surviving longer and as a result enter the public school settings with the need for dysphagia service. Research findings have indicated both the necessity and the need for swallowing services within the actual school day. Over the last decade this has evolved to treatment and management of swallowing services as part of the scope and practice of the speech language pathologist and other related professionals within the school settings. [13] reported that dysphagia school day service is an important part of the speech language pathologist's responsibility.

There have been a multitude of mandates related to health issues of children with disabilities within the school setting. There are federal laws such as [11] that require swallowing services to be provided as long as it is educationally compulsory. By the 1970 's, there was only a small number of children with disabilities who were being educated in the public school setting. There were two major federal laws that caused the education profession to have a paradigm shift: The Education for All Handicapped Children Act (EHA) and the Individuals with Disabilities Act (IDEA). EHA was established for children to have a right to public education for all regardless of a disability while IDEA require schools to provide individualized or special education for children with various qualifying disabilities. IDEA is federally mandated and was originally approved in 1975 in order to provide a free and appropriate public education to children with disabilities, and regulates the special education process in the schools. Free and appropriate public education better known as (FAPE) provides special education and related services to children with disabilities in order to promote further educational opportunities such as employment, vocational and rehabilitative services, independent living, etc. [11], [18] protect the rights of individuals with disabilities in programs and activities that receive federal financial assistance.

Section 504 provides that: "No otherwise qualified individual with a disability in the United States . . . shall, solely by reason of her or his disability, be excluded from the participation in, be denied the benefits of, or be subjected to discrimination under any program or activity receiving Federal financial assistance ..."

The U.S. Department of Education (ED) enforces Section 504 in programs and activities that receive federal funds from ED. Recipients of these funds include public school districts, institutions of higher education, and other state and local education agencies. ED has published a regulation implementing Section 504 (34 C.F.R. Part 104) and maintains an Office for Civil Rights (OCR), with 12 enforcement offices and a headquarters office in Washington, D.C., to enforce Section 504 and other civil rights laws that pertain to recipients of funds. The Section 504 regulation requires a school district to provide a "free appropriate public education" (FAPE) to each qualified person with a disability who is in the school district's jurisdiction, regardless of the nature or severity of the person's disability [18]. One way to ensure that programs meet individual needs is through the development of an individualized education program (IEP) for each student with a disability. IEPs are required for students' participation in the special education programs for all recipients of federal funding under the IDEA. Schools systems can address swallowing and feeding and provide appropriate public education through (FAPE) by developing a system wide approach that establishes a feeding plan for each student. The swallowing process in the schools should include a referral and identification process, method for assessing swallowing and feeding skills, ethnographic interview with the family, child, teacher, school staff, clinical observation of child with peers, eating, behavior, and further interaction between child and family within the home. Additionally, it must include an approach to develop a program for working collaboratively with the school cafeteria staff if further therapeutic intervention is needed for child success. Part B of IDEA require participating states to ensure that FAPE is made available to all qualified persons with disabilities. To be eligible, a child must be evaluated as having one or more of the disabilities listed in IDEA and determined to be in need of special education and related services. Evaluations must be conducted according to prescribed procedures. The disabilities specified in IDEA include: mental retardation, hearing impairments including deafness, speech or language impairments, and visual impairments including blindness, emotional disturbance, orthopedic impairments, autism, traumatic brain injury, other health impairments, specific learning disabilities, deaf blindness, and multiple disabilities. (IDEA, 2004, p.6). Based on the provisions included in the IDEA, FAPE is made available for children with swallowing difficulties in the public schools. The education is to be provided according to the child's Individualized Education Plan (IEP) [11].

The American Speech Language Hearing Association (ASHA) has developed specific guidelines for the speech language pathologists working within the public school setting. [3] developed a Scope of Practice in Speech Language 
Pathology guideline in order to better prepare and enhance the services provided to children with disabilities in the schools who are also in need of swallowing services relative to assessment, management, and therapeutic intervention. [3] highlights services that are required such as screenings, assessments, diagnoses, treatment, counseling, and referrals to individuals who have problems in the areas of oral, pharyngeal, laryngeal, and esophageal aspects of feeding or swallowing due to respiratory, pharyngeal, laryngeal, oral, neurological, or genetic deficits, or swallowing caused by neonatal difficulties. Also, it described the Knowledge and Skills Needed by SpeechLanguage Pathologists Providing Services to Individuals with Swallowing and/or Feeding Disorders [2] designed to assist the speech language pathologists in swallowing management; and included in this document was a list of competencies needed in swallowing assessment, management and intervention, professional skills, and team management that is required within the work setting. Additionally, [1] reported the development of the Guidelines for Speech-Language-Pathologists Providing Swallowing and Feeding Services in the School setting. The information included in the document explains more specifically about the role of swallowing management for the speech language pathologist, and the role of related health professionals such as the parent, classroom teacher, psychologist, nurse, physical therapist (PT), occupational therapist (OT), etc. The document has been found to be helpful for the management of dysphagia for the practicing speech language pathologists in the schools as they advocate for safety while eating in school, appropriate food utensils, food nourishment, and properly trained personnel. While there are studies that have addressed effective swallowing services in schools there is not one recipe plan for managing swallowing disorders in the school settings.

\section{Swallowing and feeding challenges and issues in the school setting}

According to [16], dysphagia is a complex syndrome that is defined as difficulty with swallowing and/or feeding function. Feeding includes the act of preparing food and getting it to the child either orally or through other alternative means. Feeding disorders include problems gathering food and getting ready to suck, chew, or swallow food. Swallowing includes the actual manipulation of food in the mouth and directing its passage from the oral cavity to the stomach. There are over a million children nationwide who are identified with severe feeding/swallowing struggles and thousands more that go undiagnosed [6]. Feeding struggles are often essential symptoms for over two hundred four diagnoses [14]. There is a general lack of awareness and understanding on how to diagnose and treat infant and children with feeding struggles that can lead to serious swallowing complications. Families are often found going from one medical professional to another.

\section{Who is at risk for swallowing in the schools?}

Swallowing disorders occur in all age groups, and can occur as a result of a variety of inherited abnormalities. Students at high risk for a swallowing disorder are those identified with: cleft palate, cerebral palsy, autism, TBI, various neurological impairments, picky eaters, nutritional deficiencies, low birth weight, ADHD, certain medications (such as diuretics, antihypertensives, and antidepressants), eating disorders, and syndromes. Recognizing signs and symptoms of swallowing are critical for identifying children with evaluation and intervention needs in this area. If multiple symptoms are present, it is imperative that the student's swallowing and feeding issues be evaluated as soon as possible. The most common signs and symptoms of swallowing disorders are:

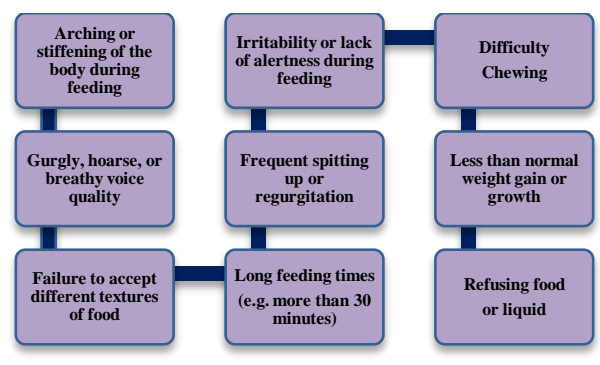

Figure 1. Signs and Symptoms of Swallowing

\section{Food preparation and guidelines for swallowing and feeding dietary modifications}

The United States Department of Agriculture (USDA) [19] regulated accommodations for special dietary needs in federally funded school nutrition programs. These regulations required substitutions and/or alternatives for modifications in meals for students with disabilities or for students who are 504 eligible [7 CFR, Sec $210.10(\mathrm{~g})$ ]. It is essential to note that dietary modifications and accommodations for those students with disabilities who have restricted diets can only be made with a documented statement signed by a licensed physician. The licensed physician statement must identify key components: 1) the child's disability and explanation as to why the disability restricts the child's diet; 2) the child's livelihood affected by the disability; 3 ) 
the foods and/or foods to be omitted from the child's diet; and 4) the foods or choice of foods to be substituted [8].

\section{The interdisciplinary team}

The purpose of the team is to provide structure and organization for addressing issues and challenges for the complexity of problems relative to swallowing and feeding disorders that may exist and arise. The members of the team: parents, speech language pathologist, classroom teacher, school nurse, psychologist, physical therapist, occupational therapist, social worker, physician, and/or community leaders. The major role of the team includes the following:

Table 1. Swallowing and feeding team assessment and management

1. Identifying children who are at risk for swallowing and
feeding disorders who already have problems and require
specialized plan of care for services.
2. Collaborating and consulting with families and their
physicians, and or nurses within the community. This includes
identification, evaluation, management, and monitoring
appropriate evidence based practice programs and services for
children and their diverse families.
3. Conduct a complex assessment approach that involves
interviews with caregiver, school staff, interdisciplinary
assessment, oral care, mealtime observation of meals at home
and school, snacks, inquire about family routines, equipment
and utensils used for eating, color and food
preferences/textures, positioning of the child, classroom
hygiene, food diary, nutritional assessment, motor/sensorimotor
skill assessment, daily feeding log ongoing, complete a
genogram of family dynamics, and cultural assessment.
4. Coordination and implementation of service delivery with
assigned roles and timelines for all.

5. Collaborating with related health professionals in designing and implementing a child's health care plan with the nurse on staff that includes emergency procedures, administration of medication and techniques for communication as deemed appropriate, and the process for informing parents of emergency situations if the need arises.

6. Training and supervised related professionals, school staff, nurses, paraprofessional, and parents on proper seating, positioning, food utensils and safe nutrition and hydration.

7. Providing information to diverse families and other health care school personnel, peers, and the community at large about swallowing and feeding disorders in order to ensure safe delivery and management of swallowing services in accordance with the child's feeding plan. Individual Education Plan (IEP), Individual Family Service Plan (IFSP), and Individualized Health Plan (IHP).

8. Team evaluation, role and responsibilities establishment of the medical team and effectiveness of the programs and services. Making the best data - based decision making for recommendations and future improvements to key administrators.

9. Documentation of training manual with signatures of trained members.

\section{Establishing swallowing competency for school based service delivery}

Swallowing specialist in the school setting are required to obtain and maintain specified training and skills in order to be service providers. The required knowledge and skills provides a framework delineating the basic competencies and roles for optimum service delivery noting age and medical condition for the child receiving the services.

The basic competencies may be developed in several ways, for example: university courses, observation of established programs, mentorship, state and national workshops to include conferences, online courses, and observation of modified barium swallow (MBS), video fluoroscopic studies (VFS) and Fiberoptic Endoscopic Evaluation of Swallowing (FEES). The recognized school based swallowing service delivery sequence must include but is not limited to: referral service, obtain permission to evaluate as needed, obtain parent permission for medical records/release, obtain physician's order when necessary or as needed for swallowing studies, provide parent/caregiver notification, swallow screening and conduct a swallow evaluation including interpretation of results to team and family, written report and recommendations to be included in the child's IEP, IFSP, and/or IHP. When a child is eligible for swallowing services additional steps are required to implement service delivery: establishment of an IEP team, development of goals and objectives appropriate to meet the child's needs, development of swallowing and feeding plan to include a food diary for two weeks, and development of an emergency plan and intervention.

\section{Cultural and linguistic diversity for swallowing and feeding consideration}

The world is constantly changing and becoming more ethnically diverse than ever before. The demographical changes are reflected in our public school system. Children and families come to school with varying styles of beliefs, behaviors, communication and lifestyle. All health professionals must be able to provide culturally appropriate services to children and families. All of us have a culture and some individuals that may mean many cultures that relates to ethnicity, race, gender, age, religion that has varying influences on the family. Culture can be defined as the rainbow of rules, practices, beliefs, ideologies, ideas of the rules of life that shapes us as the human beings that we are today. Culture shapes one's values [14]. Understanding family culture is crucial to successful outcomes for the school, child, family, and community. People from different cultural backgrounds eat different foods and what may be 
important to the school professional may not be important to the family. Culture can have many different meanings and involve many shifts in family dynamics and implementation. Culture can be referred to as family dynamics, eating environments, food preferences, food style, food preparation and method, selection of food utensils, food and family traditions, gender dynamics, food ingredients, quantity of food selection on the plate, and the types of foods eaten at different meals can vary amongst cultures. It is necessary for the school team to consider the feeding practices and preferences, beliefs at the beginning of the assessment process in order to obtain sufficient information significantly important to the child's health and nutritional needs. It is important to be aware that areas in which families live and where their ancestors originated from has an enormous impact on food likes and dislikes.

This usually results in food preferences and patterns of food choices selected within a family's cultural and/or regional group. Additionally, professionals must conduct an understanding and collection of food beliefs, religious beliefs, and food practices as part of the assessment for swallowing and feeding.

Furthermore, families still eat together though it may be in the car, at the mall, en route to soccer practice, or at a fast food restaurant. The common element of food and family is still important but may be lacking some of the cultural dimensions of the home based family meal. Food is the part of the soul and a celebration of both life and family. Food functions symbolically as a communicative practice for the family in which we share, create, and manage understanding of our cultural habits, rituals, and tradition.

\section{Management strategies for feeding and swallowing problems}

The goals of swallowing management are designed to promote adequate nutrition/hydration in addressing and developing age appropriate feeding skills. Each management technique must be customized to meet the individual needs of the population served because ultimately, treatment strategies are focused to educate, train, and work collaboratively with swallowing team members. The management strategies can be divided into two components:

1) Facilitative Strategies that promote and/or develop normal feeding skills.

2) Compensatory strategies that help to ensure successful feeding in the presence of the underlying swallowing problems.
Table 2. Facilitating Strategies for Swallowing

- Oral motor exercise management

- Oral stimulation

- Developing chewing skills

- Establishing a non-nutritive suck

- Intervention for behavioral feeding disorders

- Reducing oral aversions

- External pacing/establishing internal rhythm

Table 3. Compensatory Strategies for Swallowing

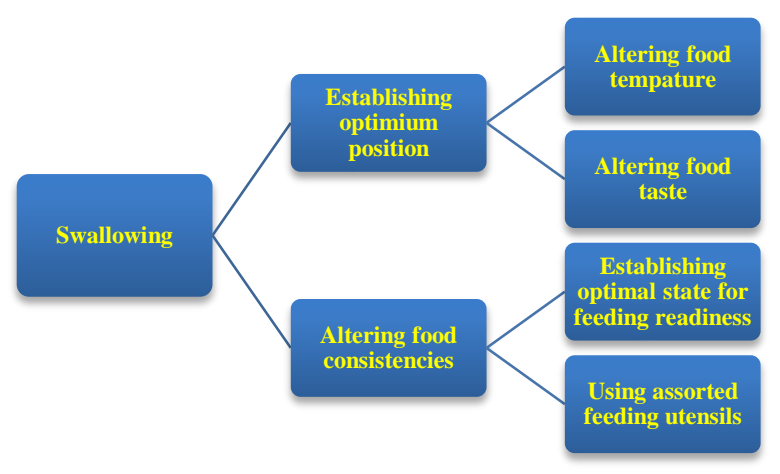

\section{Accommodations and Modifications for Children}

The Department of Education within each state has proposed guidelines as to the requirements for meal accommodations, modifications, and substitutions for children nutritional needs. The Rehabilitation Act of 1973, the Education of the Handicapped Act of 1975, and the Americans with Disabilities Act of 1990 and 2008 (ADA) state that persons with disabilities have the support of these laws that prohibit discrimination and require that children be provided free and appropriate public education. The U.S. Department of Agriculture (USDA) regulations under Title 7, Code of Federal Regulations (7 CFR), sections 15.3(b) and $210.10(\mathrm{~m})$, require substitutions or modifications in the National School Lunch Program and School Breakfast Program for children whose disabilities mandate restrict diets.

Children in today's schools need the proper nutrition in order for them to develop lifelong eating patterns that is consistent with the Dietary Guidelines for the Americans and the Food Guide Pyramid. More than half of today's children eat one in three major meals in schools and one in ten children eat two of three main meals in school [6], [8], [9]. This can be challenging at times due to food choices and influence of television and social media. According 
to [6] there were nearly 30.5 million children in today's schools being served lunches, and 19.8 million of the foods served are for free lunches with a total of five billion lunches being served annually.

There are over 90,000 schools/institutions serving school breakfasts to 14 million students daily [6].

This can vary from state to state, and from one community to the other due to regional and variations in food, labor, and variations in school equipment and school infrastructure; this is challenging to schools and most of all challenging for children with disabilities who have restricted dietary needs. It is important more than ever for schools and communities to come together and put in place effective policies and practices that should have been implemented decades ago regarding food, nutrition, and physical environments in the schools. Generations have changed; and implicitly our future well being as communities and as a nation lies with the students in our schools [6], [8], [9]. It is crucial for schools to provide the tools necessary for children to thrive and achieve in school. The quality of children's food that they eat is vital to their overall growth and developmental patterns. Also, the meals provided gives children the essential nutrients that enhances their cognitive, social, and language development abilities.

There are increasing numbers of children with complex medical and developmental needs that require evaluation and intervention in the specific areas of nutrition, diet, feeding, and swallowing. Indeed, swallowing has received a major attention in contemporary public school settings [6]. These children include an immense representation of various disorders and conditions such as: autism, ADHD, Learning disabilities, cerebral palsy, traumatic brain injury, and any other neurological or neuromuscular impairments, craniofacial anomalies, developmental disabilities, etc.

Based on these disorders, nutrition, feeding, and swallowing should be at the façade for these school programs with children who have disabilities/disorders.

In spite of the inclination of these conditions and mandates, nutrition, swallowing, and feeding practices have been inconsistently addressed across school systems; and few schools have developed written policies and procedures focused on current problems. In order to address the issues and challenges, it is useful to have ongoing interactions among school personnel, families, birth to three providers, school nurse, speech language pathologist, physical therapist, occupational therapist, social workers, school directors, physicians, nurse practitioners, physician assistants, and other medical related professionals.

\section{Physician Medical Statement for Services}

According to [6], states were permitted to expand the list of recognized medical authorities to improve access to meal accommodations for children with special dietary needs. Also, [6] reported that The Americans with Disabilities Act (ADA) 2008 made important changes to the implication and interpretation of the term disability as it relates to children and their nutritional needs.

Furthermore [6] described some important policy changes related to the requirements for school food authorities (SFAs) and Local Education Agencies (LEAs) that participate in the National School Lunch Program, School Breakfast Program, Special Mild Program for Children, and the Fresh Fruit and Vegetable Program (School Meal Programs) to provide modifications and accommodations to program meals and/or meal services in order to accommodate children with disabilities.

Additionally, SFAs have the consent to accommodate special dietary needs that do not constitute a disability, and does accommodate request associated with religious or moral convictions or personal preference. The meals offered must ensure that breakfast, lunch, and snack, or other designated meals must be obtained through the School Meal Programs (SMPs) and must meet the customary regulations for compliance.

Furthermore, the meals that are offered to all children must be available at no extra charge. In order for the SFAs to make this process successful it takes a team approach for all professionals including school officials, teachers, parents, speech language pathologists (SLP), psychologists, physical therapist (PT), occupational therapist (OT), school nurse, dietician, food service staff, school nutritionist, principal, director of special education, social worker, and any other school medical professional within the school system and/or stakeholder to come together to plan effectively for the success of all children who require specific dietary changes and modifications while in the school setting. Table 4 lists the process that must be followed in order for those actions to take place in a child's educational program.

This program also applies to private schools. The program extends out to schools that do not participate in the School Breakfast Program and funding for such a program for schools not receiving financial assistance are eligible to receive funds from non-profit school food service to account for in order to cover the cost for the accrued and mandated services in order to provide and accommodate children by the IDEA. 
Table 4. Steps to Child's Restricted Swallowing/Feeding Diet Program

1. Conduct a multidisciplinary team meeting and obtain consent for swallowing evaluation.

2. Conduct a comprehensive swallowing evaluation.

3. Conduct a family cultural feeding evaluation.

4. Conduct a food diary at home and at school if necessary as part of the planning and management for restricted dietary needs.

5. Request parents to obtain a written statement from a state licensed healthcare professional (e.g., physician, nurse practitioner, physician assistant, etc.) for a medical prescription for restricted diet.

6. Develop an IEP/IFSP/IHP

7. Design a nutritious meal plan and program for the child.

8. Team follow-up and monitor child's restricted dietary program on a 1-3-month interval in order to manage any medical complications relative to swallowing.

\section{Requiring a child's medical statement for LEAs}

The child's medical statement must include a description of the child's physical or mental health impairment. There must be an explanation provided of the child's restricted dietary needs in order to be able to effectively accommodate the disability. In addition, there should be a list of foods or foods omitted from the child's diet and food/choice of foods to be substituted for their program. Other considerations that must be a part of the program should contain mealtime feeding and/or equipment support, texture modifications for food such as chopped, pureed, grounded, minced, thickened, soft and specialized equipment and utensils, and positioning. This process is crucial in order to ensure proper and safety meal management. The planning process for meal management must take into consideration the integration of the meal program within the child's educational setting that is reasonable and appropriate in meeting the child's needs. The school food authorities and local education agencies are responsible for the accessibility of food service areas and for ensuring the successful provision for food service assistants as needed. Comprehensive management and planning for adaptive feeding equipment or assistants must also be carefully planned and included into the child's IEP/IFSP/IHP.

\section{Nutrition, Swallowing and Feeding Needs}

It is crucial for professionals providing services to children with disabilities and disorders identify some of the most critical and vital issues that are indispensable for nutritional success. Listed below in Table 5 are some suggested guidelines that must be considered as part of the design, plan, and implementation model of a successful program. Schools must be prepared to hire and conduct training in nutrition, swallowing and feeding evaluation and intervention for their personnel, families, and community. This is critical in protecting children, schools and agencies. See Table 5 Guidelines for Nutrition, Swallowing, and Feeding for Children with Disabilities and Disorders in Schools.

Table 5. Guidelines for Nutrition, Swallowing, and Feeding for Children with Disabilities and Disorders in Schools
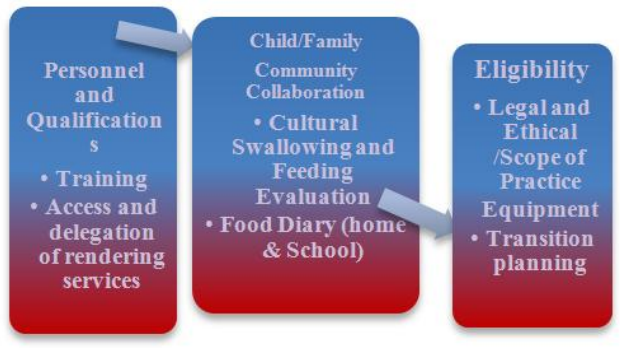

\section{Food Preparedness and Equipment Needs}

$\checkmark \quad$ Blender (Commercialized Version)

$\checkmark \quad$ Bibs and Accessories

$\checkmark \quad$ Food Processor

$\checkmark \quad$ Plate guards

$\checkmark$ Pediatric Drinking Aids and Straws/Holders

$\checkmark \quad$ Food guards for plate

$\checkmark \quad$ Various plate sizes and designs (divided, high sided, scoop dish, and/or partitioned scoop dish)

$\checkmark \quad$ Placemats and/or trays with outlines for the plate

$\checkmark \quad$ Adaptive cups with/without lids

$\checkmark$ Utensils with handles, coating on spoon, bendable versions, and textured

$\checkmark \quad$ Photos in order to stimulate and provide visual cues

$\checkmark$ Food thickeners (i.e., gel type, powdered)

$\checkmark \quad$ Pureed Foods (i.e., Heat \& Eat by Thick It, and Powders by Phagia)

$\checkmark \quad$ Pre-thickened drinks (i.e., Aquacare H2O)

Figure 2. Food Preparedness and Equipment Needs and Considerations for SFAs, LEAs, SLPs 
- Dual Cup

- Puree Food Molds

- The Go Plate

- Thick-IT

- Thick \& Easy

- Wedge Cup

- Thicken-Up

- Thik \& Clear

- Phagia Puree Mixes

- Patterson Medical

- Good Grips Adaptive Utensils

- Wedge Up

- Hormel Healthy Shots

- Boost Nutritional Pudding

Figure 3. Various companies/brands for adaptive equipment and specialized foods

As part of the swallowing evaluation it is essential for the speech language pathologist and parent(s) to work together to conduct a food diary for the child. This is necessary to determine the likes and dislikes of food, textures, smells, and environmental barriers that interrupt successful eating and feeding. [8] provided suggestions for parents and professionals to include and observe as part of the child's nutrition and swallowing program in the school and home setting. Table 6 suggested items to consider as part of the planning and management program for the child's nutritional diet.

Table 6. Food Diary Suggestions

Parent conducts a 3-5-day food diary at home and record everything
that the child consumes for over a 3-5-day period.
Time (food served each day) Is there a preference?
Location (best successful place to feed and eat)
Food Items
Determine the amount of food to be served
Drink amount/consumed
Amount of time it takes to cook and prepare food
Behaviors
Positioning
Environmental Distractions (i.e., lighting, smell, sounds) that may
interfere with eating
Behaviors notes (i.e., opens mouth, turns away, pushes food away,
leaves food on plate, refuse to eat food, cries, throws food, spits food
out of mouth)
out of mouth)

As part of the food and nutrition aspects of the child it is necessary for the speech language pathologist to conduct a nutrition and swallowing evaluation of the child at school. See Figure 4 on
Nutrition and Swallowing Evaluation for Children with Special Dietary Needs.

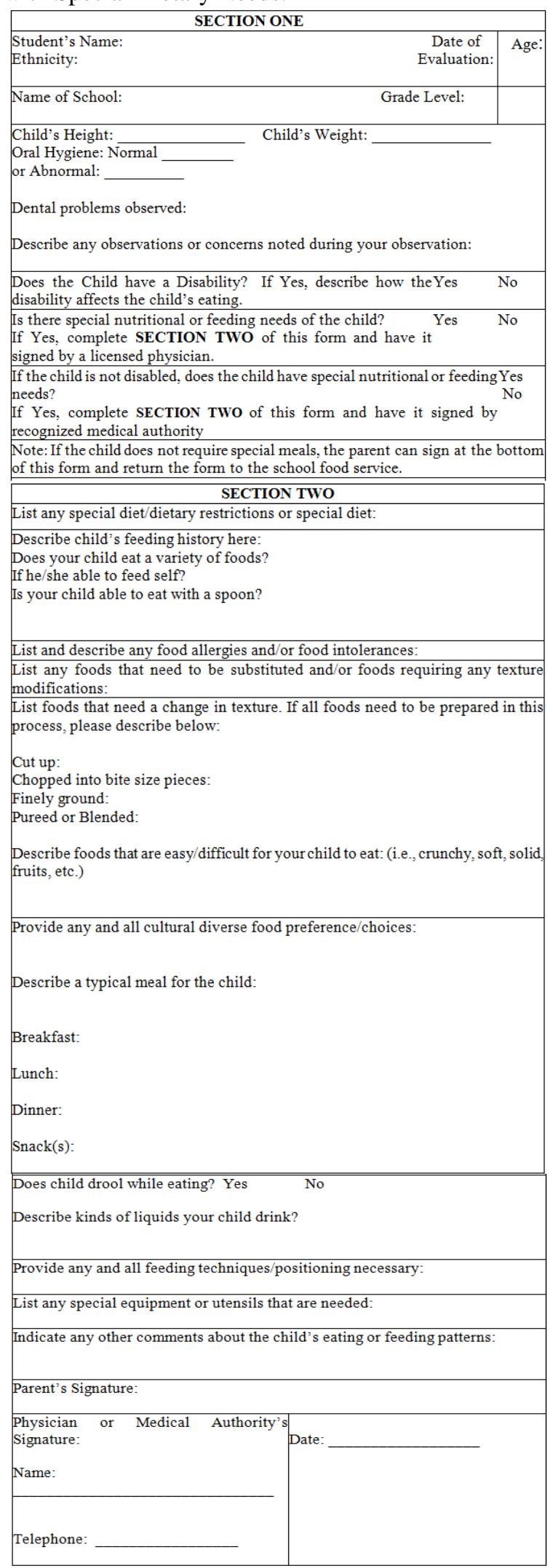

Figure 4. Nutrition and Swallowing Evaluation for Children with Special Dietary Needs 


\section{Conclusion}

As professionals we have a moral responsibility to promote healthy eating and lifestyle changes for children of all ages. Our children are faced with various eating habits throughout their entire life at such a young age. Furthermore, learning to choose and enjoy different types of foods, textures, and sensual eating in food will provide children the foundational framework for making healthy choices that merge into adulthood. Good nutrition is essential for healthy growth and brain development.

This study addressed many of the challenges and issues for professionals, parents, and children with swallowing complications in the public schools. [11], [18] have indicated the importance and responsibility for the school children to be provided the health related services that children with disabilities need.

The challenges and issues related to swallowing are complex, with the possibility for significant medical and legal risks if proper procedures are not addressed and resolved as to how to ensure safety feeding and eating within the school setting. It is most advantageous for a collaborative and/or interdisciplinary team be positioned in place in order to advocate for feeding effectively and taking the necessary steps to ensure proper nutrition, consistent feeding strategies and techniques, training for parents and staff, in order to protect the child's safety and most of all health within a dignified manner. There is continued debate as to the responsibility, identification, and categorization processes for swallowing within the school setting. Furthermore, assessment and the appropriate therapeutic procedures have compelled professionals to plan, organize, and implement the best effective swallowing program.

It is important to consider the protection of health and safety of the child and family, cultural influences that impact the selection of the appropriate feeding methods and foods chosen, development of the appropriate goals and objectives, delegation and supervision of professionals and parents, building/classroom emergency procedures, space and equipment, obligations and responsibility/liability in order to meet the needs of children and families in the school setting. School systems have to begin organizing and planning for a nationwide model for swallowing within the school setting. Further research is warranted to meet the needs of children and families as children and types of disabilities are on the rise.

There are existing and future challenges for future speech language pathologist, educators, administrators, nursing professionals, and children and their families in dealing with swallowing issues. This study provided information and guidelines for addressing swallowing challenges and issues in the school setting. It is beneficial for children, families, and the medical community to come together in order to meet the needs of the children. It is crucial for parents to be involved and included on the team as they are able to provide information on nutrition, medical complexities if any, and the educational needs. School systems can no longer not consider the educational suitability for meeting the needs of swallowing within the school setting. Future research is necessary in order to develop a national model for swallowing that would be coherent across all educational school systems in order to better prepare and meet the needs of children and families with disabilities.

\section{References}

[1] American Speech-Language-Hearing Association. (2007). Guidelines for speech- language pathologists providing swallowing and feeding services in schools. Available from http://www.asha.org/policy. (Access date: 15 June 2016).

[2] American Speech-Language-Hearing Association. (2002). "Knowledge and skills needed by speech language pathologists providing services to individuals with swallowing and/or feeding disorders". Available from http://www.asha.org/policy. (Access date: 15 June 2016).

[3] American Speech-Language-Hearing Association. (2015). "Scope of Practice in Speech Language Pathology". Available from http://www.asha.org/policy. (Access date: 15 June 2016)

[4] American Speech-Language-Hearing Association. (2008). "2008 Schools Survey report: Caseload characteristics". Available from: http://www.asha.org/ policy. (Access date: 15 June 2016).

[5] Arvedson, J. (2008). "Assessment of Pediatric Dysphagia and Feeding Disorders: Clinical and Instrumental Approaches". Developmental Disabilities Research Reviews, 14, 118-127.

[6] Enwefa, R., Enwefa, S., (2016) "Dysphagia management in the school setting". Paper presented at the Mississippi Speech Language Hearing Association Annual Continuing Education Conference, Jackson, MS.

[7] Enwefa, S., Enwefa, R. and Rey, M (2014a) “Oral care in prevention of aspiration pneumonia in dysphagia patients". Paper presented at the Mississippi Speech Language Hearing Association Annual Continuing Education Conference, Jackson, MS.

[8] Enwefa, R, Enwefa, S., and Rey, M (2014b) "Mealtime challenges for children with autism spectrum disorders and sensory processing disorders". Paper presented at the Mississippi Speech Language Hearing Association Annual Continuing Education Conference, Jackson, MS

[9] Enwefa, S., Enwefa, R. (2013) "Comprehensive dysphagia patient management: Assessment, Nutrition, and Medication Challenges for the Speech Language Pathologist". Paper presented at the Mississippi Speech 
Language Hearing Association Annual Continuing Education Conference, Jackson, MS.

[10] Enwefa, R., Enwefa, S., Nyarambi, A. (2012) "Speech-Language, Nutrition and Behavior Therapy for Autism Spectrum Disorders". Paper presented at the Mississippi Speech Language Hearing Association Annual Continuing Education Conference, Jackson, MS.

[11] "Individuals with Disabilities Education Improvement Act of 2004" (IDEA). (December 2004). Retrieved: http: www/nichcy.org/Laws/IDEA/Documents/PL108-446.pdf. (Access date: 15 June 2016).

[12] Lefton-Greif, M. (2001). "Ethical decision making for infants and children with dysphagia". Swallowing and Swallowing Disorders (Dysphagia), 10(2), 27-30.

[13] Logemann, J.A., \& O'Toole, T.J. (2000). "Identification and management of dysphagia in the public schools". Language, Speech, and Hearing Services in Schools, 31, 26- 27.

[14] Jaballas E, Clark-Ott D, Clasen C, Stolfi A, Urban M: "Parents' perceptions of their children's weight, eating habits, and physical activities at home and at school". Journal of Pediatric Health Care 2011, 25:294-301.

[15] Lynch, E., W., \& Hanson, M. J. (2004). “Developing cross-cultural competence: A guide for working with children and their families" (3rd ed.). Baltimore: Brookes.

[16] Miller, R. M., \& Groher, M.E. (1993). "Speechlanguage pathology and dysphagia: A brief historical perspective". Dysphagia 8, 180-184.

[17] Murry, T., \& Carrau, R.L. (2009). "Clinical management of swallowing disorders". San Diego: Plural Publishing.

[18] O'Toole, T. (2000). "Legal, ethical, and financial aspects of providing services to children with swallowing disorders in the public schools". Language, Speech, and Hearing Services in Schools, 31, 56-61.

[19] "Rehabilitation Act of 1973, Section 504", 29 U.S.C. Ch $16 \S 701(b)(1)$.

[20] U.S. Department of Agriculture. (2001). "Accommodating children with special dietary needs in school nutrition programs: Guidance for school food service staff'. Available from http://www.fns.usda.gov/ cnd/guidance. (Access date: 15 June 2016). 